\title{
Contribuições dos objetos de aprendizagem para ensejar o desenvolvimento do pensamento crítico nos estudantes nas aulas de Física
}

\section{Contributions of learning objects give to rise the development of critical thinking in students in Physics classes}

Ana Marli Bulegon ${ }^{1}$ • Liane Margarida Rockenbach Tarouco ${ }^{2}$

\begin{abstract}
Resumo: No intuito de analisar as contribuições dos objetos de aprendizagem (OA) para ensejar o desenvolvimento do pensamento crítico nos estudantes a partir de aulas de Física, elaboramos um conjunto de atividades de aprendizagem com o uso de OA referente ao conteúdo de Termodinâmica. As atividades foram organizadas no modelo metodológico dos Três Momentos Pedagógicos (TMP) de acordo com o Ciclo de Kolb. Essas foram encapsuladas no eXe Learning e disponibilizadas no ambiente virtual de aprendizagem Moodle aos estudantes de Ensino Médio de uma escola estadual do Rio Grande do Sul. Os resultados deste trabalho indicam que o uso dos $\mathrm{OA}$, inseridos nas atividades de aprendizagem, contribuíram para o desenvolvimento de habilidades de pensamento crítico, como: atenção, observação, avaliação, análise crítica, justificativa, importância, utilidade prática, síntese, entre outras.
\end{abstract}

Palavras-chave: Objetos de aprendizagem. Pensamento crítico. Ensino de física. Atividades de aprendizagem.

\begin{abstract}
In order to analyze the contributions of learning objects (LO) to raise the development of critical thinking in students in physics classes, we have developed a set of learning activities with the use of LO about the content of Thermodynamics. The activities were organized in the methodological model of the Three Pedagogical Moments (TMP) according to the cycle of Kolb. These were encapsulated in eXe Learning and available in Moodle to high school students in a public school in Rio Grande do Sul. The results of this study indicate that the use of LOs, inserted in learning activities, have contributed to the development of critical thinking skills such as attention, observation, evaluation, critical analysis, justification, importance, practical utility, synthesis, among others.
\end{abstract}

Keywords: Learning objects. Critical thinking, Learning activities. Physics teaching.

\footnotetext{
${ }^{1}$ Centro Universitário Franciscano (UNIFRA), Rua Silva Jardim, 1175, Centro, CEP 97010-040, Santa Maria, RS, Brasil. E-mail: <anabulegon@gmail.com>

${ }^{2}$ Faculdade de Educação, Universidade Federal do Rio Grande do Sul, Porto Alegre, RS, Brasil.
} 


\section{Introdução}

As rápidas mudanças da ciência e tecnologia, na sociedade atual, contribuíram para que a comunicação seja facilitada por equipamentos modernos e sofisticados que aproximam pessoas de todo o mundo. A rapidez com que as informações e notícias são transmitidas exige, cada vez mais, que as pessoas sejam capazes de pesquisar, questionar, que tenham iniciativa e sejam capazes de solucionar problemas. Isto é, que saibam realizar suas tarefas com competência, que saibam tomar decisões e intervir no meio em que vivem de forma a contribuírem para a melhoria da qualidade de vida. Tais habilidades necessitam que as pessoas tenham desenvolvido e façam uso de capacidades de pensamento crítico. Essas capacidades permitem, ao indivíduo, resolver problemas e tomar decisões racionais (HALPERN, 1999). Diante dessas constatações, pensar criticamente, enquanto "[...] uma forma de pensamento racional, reflexivo, focado naquilo que se deve acreditar e fazer" (ENNIS, 1985, p. 46), ou seja, um tipo de pensamento envolvido na resolução de problemas, formulação de hipóteses e inferências e tomada de decisões (ENNIS, 1985; MANDERNACH et al., 2009), e não apenas baseado em sua crença sobre aquilo que deve ou não fazer, tornou-se uma meta educacional, e traz novas possibilidades, desafios e incertezas ao processo de ensino e de aprendizagem.

A Lei de Diretrizes e Bases da Educação Nacional (LDB) (BRASIL, 1996) contemplou essas exigências e estabeleceu uma reformulação no Ensino Médio no país, no sentido de indicar caminhos e fornecer propostas para a melhoria do ensino. Algumas finalidades para o Ensino Médio, apontadas por esta lei, em seu Art. 35, merecem destaque, como os itens III e IV, citados a seguir:

[...] III - o aprimoramento do educando como pessoa humana, incluindo a formação ética e o desenvolvimento da autonomia intelectual e do pensamento crítico;

IV - a compreensão dos fundamentos científico-tecnológicos dos processos produtivos, relacionando a teoria com a prática, no ensino de cada disciplina. [...] (BRASIL, 1996, p. 15).

Pode-se verificar que o pensamento crítico é apontado como um objetivo comum de várias áreas do conhecimento da Educação Básica. Segundo Vieira e Vieira (2003), um dos desafios do ensino é tornar o educando uma pessoa crítica e atenta aos fenômenos e técnicas do seu cotidiano.

$\mathrm{Na}$ disciplina de Física, Fiolhais e Trindade (2003) apontam que alguns estudantes apresentam dificuldades na aprendizagem de alguns conceitos, manifestadas: na dificuldade de resolução dos problemas propostos, na pobreza conceitual, na falta de contextualização e na incapacidade de aplicar os conceitos estudados em situações do cotidiano. Engajar os estudantes na construção de seu próprio conhecimento e relacionar a teoria, estudada nos manuais didáticos, com o cotidiano, é uma das maneiras apontadas pelos pesquisadores para diminuir esse problema e desenvolver uma compreensão maior dos fundamentos teóricos que embasam os conhecimentos escolares (MANDERNACH et al., 2009; SCHON, 2000; SENDAG; ODABASI, 2009). Para que os estudantes consigam confrontar os significados existentes em sua estrutura cognitiva com os novos, e tomar parte de um diálogo que contribua com sua aprendizagem, 
algumas condições, que vão além de um método ou estratégia de ensino e de aprendizagem, são necessárias. Uma dessas condições é a ocorrência do pensamento crítico.

Para estimular o desenvolvimento do pensamento crítico, os Parâmetros Curriculares Nacionais (PCN) (BRASIL, 2000) para o Ensino Médio sugerem que, no ensino de Física, as questões para discussão devem ser centradas nos estudantes, contextualizadas e de interesse deles. Isto é, que seja desenvolvida "Uma Física cujo significado o estudante possa perceber no momento em que aprende, e não em um momento posterior ao aprendizado" (BRASIL, 2000, p. 23).

A referência pedagógica apontada pelos PCN está voltada para a preparação de jovens, não apenas para que eles, simplesmente, conheçam a Física, mas para que esse conhecimento possa auxiliá-los a pensar, agir e interferir em situações de seu cotidiano, ou seja, tornar os jovens capazes de lidar com situações reais no momento em que elas acontecem, como: crises de energia, problemas ambientais, manuais de aparelhos elétricos e eletrônicos, exames médicos, notícias de jornal, e assim em diante. Para que essas finalidades sejam atingidas, é necessário desenvolver, nos estudantes, as habilidades de pensamento crítico, como: observar, experimentar, investigar, interpretar, reconhecer, construir, entre outras. Manter a atenção ao desenvolvimento dessas habilidades, ao longo das atividades de aprendizagem, poderá promover, nos estudantes, sua aprendizagem segundo os PCN (BRASIL, 2000).

Entretanto, fazer a transposição entre o conhecimento escolar e o conhecimento cotidiano exige do educador um conhecimento não só da Física, mas de métodos e estratégias de ensino que sejam adequadas para desenvolver tais significados e habilidades. O ensino de Física ministrado nas escolas brasileiras, por sua vez, ainda se resume ao uso de leis e algoritmos utilizados nos livros didáticos, com pouca ou nenhuma relação com o cotidiano dos estudantes (ASSIS, 2001; DAMASIO; STEFFANI, 2008). Para atenuar esta dicotomia entre teoria e aplicação do conhecimento, algumas propostas de estratégias de ensino e de recursos didáticos têm sido propostas, tais como: o ensino baseado na investigação, envolvendo a utilização do computador e, em especial, o uso de objetos de aprendizagem (OA). A representação virtual tem sido apontada como uma possibilidade de substituir ou complementar as demonstrações e experimentações (FIOLHAIS; TRINDADE, 2003) usadas no ensino de Física. Os OA, definidos como sendo um recurso pedagógico que serve para apoiar o ensino (BECK, 2001; TAROUCO et al., 2004; WELLER; PEGLER; MASON, 2003), possuem um grande potencial na área educacional para apoiar a criação, manipulação, armazenamento e pesquisa de conteúdos, facilitando a criação de analogias e de representações de situações reais. Nesta perspectiva, este trabalho apresenta uma investigação e análise do seguinte problema: quais as potencialidades e contribuições dos Objetos de Aprendizagem (OA) no desenvolvimento do pensamento crítico no ensino de Física? Uma das hipóteses de resolução desse problema é a de que os OA, inseridos em um conjunto de atividades de aprendizagem, planejadas pelo professor, como: disparador/instigador de dúvidas e questões; reforço/aplicação de conhecimentos, interligados em um plano que leve à metacognição, seriam capazes de ensejar a aprendizagem significativa e o pensamento crítico em Física. Para o desenvolvimento dessa investigação foi delineado um experimento que incluiu a elaboração de um módulo didático usando OA, com 10 (dez) aulas sobre o conteúdo de Termodinâmica. A análise da ocorrência de desenvolvimento do pensamento crítico utilizou indicadores de desenvolvimento do pensamento crítico tais como propostos por Newman, Webb e Cochrane (1995). 
Este trabalho apresenta, inicialmente, alguns pressupostos teóricos que embasaram o trabalho e, a seguir, a metodologia de trabalho, com a organização da atividade didática. A análise e discussão dos resultados obtidos, bem como as evidências de desenvolvimento de pensamento crítico são relatados em sequência, assim como as conclusões e considerações finais.

\section{Pensamento Crítico}

O pensamento crítico, para Jonassen (1996b), é o desenvolvimento de habilidades de avaliação, análise e o relacionamento entre as informações que estão sendo analisadas. Envolvem a implicação crítica, a discussão e uma reorganização dinâmica do conhecimento de forma significativa e útil (JONASSEN, 1996a), que têm um papel crucial na ativação de resolução de problemas e nos processos de tomada de decisão. Essas habilidades podem ser utilizadas para: definir problemas, tomar medidas no sentido de um objetivo, tomar decisões e realizar avaliações retrospectivas (JONASSEN, 1996b; KAYA, 1997; SCHON, 2000; SENDAG; ODABASI, 2009). Trata-se, portanto, de desenvolver habilidades gerais como: avaliar, analisar e conectar informações. Avaliar envolve fazer julgamentos, reconhecer e usar critérios em diferentes dimensões, e não expressar uma atitude pessoal sobre algo. Analisar envolve separar o todo das informações em partes significativas e compreender as inter-relações entre elas. Conectar envolve as relações entre as informações que estão sendo analisadas. Entretanto, conforme Sumner (1906), o pensamento crítico é uma questão de hábito, ou seja, é o pensamento cujo foco está no desenvolvimento: da busca da verdade, da mente aberta, de ser sistemático, analítico, curioso, confiante no raciocínio e prudente na tomada de decisões. É o tipo de pensamento envolvido na resolução de problemas, formulação de hipóteses e inferências, e na tomada de decisões (MANDERNACH et al., 2009).

Constata-se que não há uma única definição em relação ao pensamento crítico, porém, observa-se que o ponto de partida da maioria deles é a solução de um problema. O problema é o elemento disparador que pode levar ao pensar criticamente, entendido aqui como habilidade de interpretar, analisar, avaliar e criar ideias, raciocínios e argumentos de forma clara e precisa. $\mathrm{O}$ desenvolvimento do pensamento crítico exige um processo de contextualização reflexiva e, para isso, o ensino deve estar centrado na busca por questões/problemas que envolvam o cotidiano e a cultura dos estudantes. Diante disso, o papel do professor deve ser de orientador, no sentido de indicar o melhor caminho para a busca de informações na construção do conhecimento.

\section{Desenvolvimento do Pensamento Crítico}

Uma das maneiras mais eficazes de desenvolver o pensamento crítico é por meio de atividades de resolução de problemas (SENDAG; ODABASI, 2009) e de atividades práticas (SCHON, 2000). A interatividade entre professor e estudantes e, entre estudantes, também é apontada como uma maneira eficaz de desenvolver o pensamento crítico (MANDERNACH et al., 2009; SCHON, 2000). Com base nisso, foi possível delinear uma estratégia para o desenvolvimento do conhecimento como um processo reflexivo por meio da investigação e da construção de alternativas para responder aos problemas ocorridos em contextos reais. Uma 
alternativa, proporcionada pela tecnologia atual, é usar objetos de aprendizagem (OA), que permitem criar analogias virtuais de situações reais, as quais proporcionam, aos estudantes, a possibilidade de manipulação de alguns parâmetros e o teste de hipóteses inerentes à prática de atividades do mundo real (SCHON, 2000).

As interações espontâneas podem desenvolver o pensamento crítico, porém, as pesquisas têm apontado que as abordagens de ensino que envolvem a aprendizagem ativa e que são centradas nos estudantes apresentam resultados mais eficazes (MANDERNACH et al., 2009; SCHON, 2000). As etapas que envolvem o processo de simulação de um fato/fenômeno, por exemplo, reforçam ainda mais o pensamento crítico e autônomo de construção do conhecimento, pois nelas há interação do estudante com o objeto de estudo (SALIÉS, 2008). Assim, o desenvolvimento do pensamento crítico não depende do ambiente da sala de aula, do professor e nem do conhecimento dos estudantes, mas está diretamente relacionado com a interação dos estudantes com o objeto de estudo (MANDERNACH et al., 2009; SCHON, 2000), pois os estudantes de aulas online mostraram-se mais críticos do que os de aulas presenciais (MANDERNACH et al., 2009). Em uma sala de aula presencial, a interatividade é, inicialmente, social, e uma função da presença física do professor. Para que as atividades de aprendizagem se tornem interativas, as instruções presenciais requerem menos orientações do que em atividades online. Entretanto, para que haja maior interatividade nas atividades online, o professor deve elaborá-las de modo a proporcionar um diálogo entre os estudantes. Neste sentido, em aulas online, é importante que o professor estimule e incentive o debate (MANDERNACH et al., 2009). Conforme Schon (2000), os estudantes precisam ser capazes de tomar parte de um diálogo para que possam aprender o significado das operações envolvidas na resolução dos problemas, executando-as na prática para desenvolver habilidades de pensamento crítico. Para avaliar o desenvolvimento do pensamento crítico, necessita-se fazer uso de indicadores que possam ser constatados numa fase inicial e numa fase final. No presente trabalho, fez-se uso dos indicadores de pensamento crítico elencados por Newman, Webb e Cochrane (1995), tal como será descrito na seção seguinte (Quadro 1).

\section{Indicadores de Pensamento Crítico}

Newman, Webb e Cochrane (1995), ao analisarem a comunicação mediada por computador, propuseram um modelo de análise de conteúdo a partir de indicadores positivos $(+)$ e negativos (-) (que denotassem a presença ou ausência de pensamento crítico, respectivamente). Os indicadores propostos por eles (Quadro 1) estão relacionados a algumas habilidades necessárias ao desenvolvimento do pensamento crítico, como: conhecimento/experiência, novidade, relevância, importância, avaliação crítica, ambiguidades, associação de ideias, justificativa, utilidade prática-avaliação do conhecimento, e extensão da compreensão.

Esses indicadores foram escolhidos, pois eles apresentam a maioria das habilidades apontadas por Newman, Webb e Cochrane (1995) como manifestações do pensamento crítico. A investigação relatada neste trabalho buscou elicitar a ocorrência de pensamento crítico a partir da presença desses indicadores nas manifestações dos estudantes, em um momento inicial do desenvolvimento da unidade de aprendizagem e após o uso de OA, tal como a sessão seguinte descreve. 
Quadro 1. Indicadores de Pensamento Crítico

\begin{tabular}{|c|c|c|}
\hline Indicador & Sigla & Exemplo de manifestação que permite constatar o indicador \\
\hline $\begin{array}{l}\text { Conhecimento/ } \\
\text { Experiência }\end{array}$ & $\mathrm{C} / \mathrm{E}+$ & $\begin{array}{l}\text { Recorrer à experiência pessoal. Referir ao material do curso. Usar } \\
\text { material relevante de fora. Evidenciar o uso de conhecimento prévio. } \\
\text { Descartar tentativas de trazer conhecimentos externos. } \\
\text { Apegar-se a preconceitos ou suposições (pressupostos). }\end{array}$ \\
\hline Novidade & $\mathrm{N}+$ & $\begin{array}{l}\text { Novas informações relacionadas ao problema. Novas ideias para } \\
\text { discussão. Novas soluções para os problemas. } \\
\text { Repetir o que já foi fito. Pistas falsas ou triviais (insignificantes). } \\
\text { Aceitar a primeira solução oferecida. Esperar pela condução do } \\
\text { professor. }\end{array}$ \\
\hline Relevância & $\begin{array}{l}\mathrm{R}+ \\
\mathrm{R}-\end{array}$ & $\begin{array}{l}\text { Depoimentos (afirmações/avaliações) relevantes. } \\
\text { Depoimentos (avaliações) irrelevantes, distrações, desvios. }\end{array}$ \\
\hline Importância & $\begin{array}{l}\mathrm{I}+ \\
\mathrm{I}-\end{array}$ & $\begin{array}{l}\text { Pontos/Questões/Assuntos importantes. } \\
\text { Pontos/Questões/Assuntos não importantes, triviais, insignificantes. }\end{array}$ \\
\hline Avaliação Crítica & AC+ & $\begin{array}{l}\text { Avaliação/Diagnóstico crítico de contribuições próprias ou de outras } \\
\text { pessoas. Aberto a uma avaliação crítica. } \\
\text { Aceitar sem crítica ou rejeitar sem razão. Aceitar de forma não } \\
\text { crítica. }\end{array}$ \\
\hline Ambiguidades & A- & $\begin{array}{l}\text { Afirmações não ambíguas (ser claro, inequívoco). } \\
\text { Discutir as ambiguidades para clareá-las (esclarecê-las). } \\
\text { Afirmações confusas. Continuar ignorando as ambiguidades. }\end{array}$ \\
\hline $\begin{array}{l}\text { Associação de } \\
\text { Ideias }\end{array}$ & $\mathrm{AI}+$ & $\begin{array}{l}\text { Relaciona, compara, associa fatos, ideias, noções. } \\
\text { Gerar novos dados a partir das informações coletadas. } \\
\text { Repete informações sem fazer inferências ou oferecer uma } \\
\text { interpretação. }\end{array}$ \\
\hline Justificativa & $\begin{array}{l}\mathrm{J}+ \\
\mathrm{J}-\end{array}$ & $\begin{array}{l}\text { Prover/Oferecer/Fornecer provas ou exemplos. } \\
\text { Justificar soluções ou julgamentos. } \\
\text { Questões ou exemplos obscuros ou irrelevantes. } \\
\text { Oferecer julgamentos ou soluções sem explicações ou justificativas. } \\
\text { Oferecer várias soluções sem sugerir qual a mais apropriada. }\end{array}$ \\
\hline $\begin{array}{l}\text { Utilidade Prática e } \\
\text { Avaliação do } \\
\text { Conhecimento }\end{array}$ & $\begin{array}{l}\mathrm{UP}+ \\
\mathrm{UP}-\end{array}$ & $\begin{array}{l}\text { Relacionar possíveis soluções a situações familiares. } \\
\text { Discutir a utilidade prática das novas ideias. } \\
\text { Discute sem propor solução. Sugere soluções não práticas. }\end{array}$ \\
\hline $\begin{array}{l}\text { Extensão da } \\
\text { compreensão }\end{array}$ & $\begin{array}{l}\mathrm{EX}+ \\
\mathrm{EX}-\end{array}$ & $\begin{array}{l}\text { Discussão ampla. Utiliza estratégias de intervenção de amplo alcance. } \\
\text { Discussão limitada, em pedaços, fragmentada. Intervenções fracas, } \\
\text { parciais. }\end{array}$ \\
\hline
\end{tabular}

Fonte: Newman, Webb e Cochrane (1995, p. 56). 


\section{Estratégia tecnológica utilizada}

Os recursos da informática têm contribuído de forma significativa, no ensino de Física, para a modernização e democratização, sobretudo por baratear custos em áreas importantes, como as experimentações e simulações. Segundo Rosa (1995), são grandes as potencialidades do uso dos computadores no ensino de Física: coleta e análise de dados em tempo real; simulação de fenômenos físicos; instrução assistida por computador; administração escolar e estudo de processos cognitivos por meio da análise da interação dos estudantes. Para Fiolhais e Trindade (2003), o computador é utilizado no ensino de Física para: aquisição de dados por computador; modelagem e simulação; materiais multimídia; realidade virtual; busca de informações na Internet. Segundo Tarouco et al. (2004), a tecnologia da informática e comunicação permite criar material didático interativo, usando multimídia, que torna mais efetivos os ambientes de ensino e aprendizagem, aumenta a motivação e interesse do estudantes, criando oportunidades para uma participação mais ativa destes no processo de aprendizagem. A interação proporcionada pelo uso do computador e da comunicação via Internet permite utilizar diversos tipos de ferramentas, inclusive, de autoria. Jonassen (1996b, p. 83) refere esses recursos como ferramentas cognitivas, e destaca seu potencial para a construção de conhecimento, salientando seu valor como "elemento que pode levar a pensar profundamente", e que facilitam os processos de criação de significado. Para esse autor, "aprender com as ferramentas cognitivas depende do comprometimento total da mente dos estudantes nas tarefas proporcionadas por estas ferramentas, e os estudantes trabalham com a tecnologia do computador, ao invés de serem controlados por ela" (JONASSEN, 1996b, p. 83). Essa abordagem de ensino torna a atividade de aprendizagem centrada nos sujeitos envolvidos no processo de aprendizagem. Trata-se de uma proposta que envolve, também, o estimular do trabalho colaborativo, criativo, e o pensamento crítico, no qual as tecnologias provindas da informática servem de ambiente mediador para o processo de ensino e de aprendizagem.

$\mathrm{Na}$ investigação ora relatada, a estratégia utilizada envolveu o uso de $\mathrm{OA}$, pois esses têm um grande potencial de interação; suportam um alto nível de abstração, diferentemente, mas não mais importante do que as demonstrações e experimentações de laboratório, o que pode facilitar a compreensão de conceitos físicos complexos sem demandar custos para sua aquisição e manutenção. O uso dos OA visa: despertar a curiosidade e o interesse no tema específico, buscar o aprimoramento da aprendizagem e o uso adequado dos meios tecnológicos disponíveis, e tornar o estudante um participante ativo na construção do conhecimento, ou seja, contribuir com o desenvolvimento do pensamento crítico e da autonomia dos sujeitos que dele fazem uso.

Existem, atualmente, diversos repositórios de objetos de aprendizagem voltados para o ensino e aprendizagem de Física que contêm materiais educacionais digitais de tipo bastante diversificado, como: simulação, áudio, experimento, hipertextos, imagens, softwares educacionais, vídeos, questionário, slide, figura, gráfico, entre outros. Os OA do tipo simulações/animações (8 trabalhos) e a modelagem computacional (5 trabalhos) são os tipos mais citados nas publicações nacionais, nesta última década, nas revistas especializadas em ensino de Física, como: A Física na escola (2004-2006), Caderno Brasileiro de Ensino de Física (2004-2009), Ciência \& Educação (2004-2009), Investigações em Ensino de Ciências (2005-2008), Revista Brasileira de Ensino de Física (2004-2009). De acordo com os trabalhos publicados nessas revistas, a hipermídia, que agrega hipertexto e multimídia, e a câmera digital, têm merecido destaque por 
serem recursos inovadores e atuais e potencializarem a autoria e a autonomia dos mesmos nessa produção do conhecimento. Isso não exclui o trabalho com os OA do tipo exercício, leitura de texto, questionário, gráfico, tabela, imagens, figuras e slides, pois esses se mostram igualmente importantes, dependendo da estratégia pedagógica planejada.

Os objetos de aprendizagem que podem favorecer o desenvolvimento do pensamento crítico devem ensejar o desenvolvimento de uma aprendizagem ativa, ou seja, devem promover a interação entre os estudantes e o objeto de estudo, ou entre os próprios estudantes (MANDERNACH et al., 2009; SCHON, 2000); e, para isso, devem trabalhar com o ensino baseado na resolução de problemas e envolver atividades que sejam desenvolvidas pelos estudantes de forma colaborativa (SENDAG; ODABASI, 2009).

\section{Atividades de aprendizagem desenvolvidas}

Para verificar se os objetos de aprendizagem ensejam o desenvolvimento do pensamento crítico, a pesquisa aqui relatada pode ser definida como uma pesquisa qualitativa e quantitativa, visto que o problema é complexo, de natureza social (MINAYO; SANCHES, 1993; TRIVIÑOS, 1995). A pesquisa é relatada a seguir:

\section{a) Objeto de estudo}

No ensino de Física, um dos assuntos que se mostram importantes para o cotidiano dos estudantes é a Termodinâmica. A grande emissão de carbono na atmosfera, pela queima dos combustíveis fósseis, tomou conta dos noticiários de todo o mundo, e tem sido apontada como um dos grandes vilões do aquecimento global. $\mathrm{O}$ uso de bicombustíveis é uma das alternativas apontadas para minimizar esse problema. Para que os jovens, que estudam no Ensino Médio, compreendam esse processo e possam tomar decisões e intervir de forma positiva a esse respeito precisam aprender, de forma significativa e crítica, tópicos de Termodinâmica. Entretanto, ter uma visão microscópica do processo de transformação da energia dos bicombustíveis em trabalho termodinâmico (conteúdo de Física do Ensino Médio) é algo inviável nos laboratórios didáticos das escolas de Ensino Médio, devido à precariedade de equipamentos que eles possuem. Neste sentido, foram planejadas e desenvolvidas atividades de aprendizagem com o uso de OA do tipo texto, vídeo, questionário, simulação e testes. Esses tipos de OA foram selecionados por melhor se adequarem aos objetivos propostos pelas atividades e ao momento pedagógico.

Essas atividades de aprendizagem foram agrupadas em Módulos Didáticos (MD), encapsulados mediante o uso de uma ferramenta de autoria, de acesso aberto e licença do tipo Creative Commons, chamada eXe Learning ${ }^{3}$. Esta permite encapsular os materiais de forma padronizada usando o padrão SCORM (Sharable Content Object Reference Model). Os objetos de aprendizagem, assim criados, foram disponibilizados para os estudantes no Moodle enquanto Ambiente Virtual de Aprendizagem (AVA). Os módulos foram planejados de acordo com a

\footnotetext{
${ }^{3}$ Disponível em: <http://exelearning.org/wiki>. Acesso em: 06 maio 2015.
} 
metodologia dos Três Momentos Pedagógicos (TMP) ${ }^{4}$ (DELIZOICOV; ANGOTTI, 1991), e organizadas de acordo com o Ciclo de Kolb ${ }^{5}$ (KOLB; FRY, 1975).

$\mathrm{Na}$ metodologia dos TMP, pode-se desenvolver atividades de aprendizagem com o uso de diferentes recursos como os OA. Esses podem ser utilizados em qualquer um dos TMP, em consonância com os critérios estabelecidos para adequação ao modelo metodológico adotado. Para essa adequação, fez-se uso do modelo de aprendizagem de Kolb ou Ciclo de Kolb (KOLB; FRY, 1975). Para esses autores, existem quatro diferentes estilos de aprendizagem, que são: Ativos, Reflexivos, Teóricos e Pragmáticos. Neste sentido, para ensejar o desenvolvimento do pensamento crítico, as atividades de aprendizagem precisam ser planejadas de forma a proporcionar um conflito cognitivo e atingir o maior número de estilos de aprendizagem. O Quadro 2 apresenta a organização das atividades de aprendizagem, os tipos de OA utilizados e seus respectivos objetivos, em cada etapa da metodologia adotada.

$\mathrm{Na} \mathrm{PI}$, aplicou-se um OA do tipo questionário, cujas respostas foram debatidas em um fórum, no Moodle, com o objetivo de levar os estudantes a relatarem/lembrarem experiências concretas de suas vidas, o que para Kolb é a etapa da experiência concreta. Na OC, os objetos de aprendizagem escolhidos (vídeo, texto, simulação) tiveram a função de despertar, nos estudantes, as observações e reflexões necessárias à construção do conhecimento abstrato. É a etapa da observação reflexiva e da conceitualização no ciclo de Kolb. Os textos utilizados foram destacados de livros didáticos e abordam o tema Máquinas Térmicas. A atividade de aprendizagem realizada nesta etapa é apresentada no Quadro 3.

Os vídeos utilizados têm como objetivo realizar as conceituações sobre Máquinas Térmicas e Revolução Industrial, e identificar as variáveis presentes nesses assuntos. Para verificar se os estudantes apresentavam indícios do desenvolvimento do pensamento crítico, de acordo com os indicadores de Newman, Webb e Cochrane (1995), na aula seguinte, realizou-se um chat. A atividade de aprendizagem com uso de uma simulação ${ }^{6}$ teve por objetivo resgatar as concepções prévias e reflexões dos estudantes sobre Entropia.

\footnotetext{
${ }^{4}$ A metodologia dos Três Momentos Pedagógicos (TMP) baseia-se nas dimensões dialógica e problematizadora do processo educativo proposto por Paulo Freire, e estrutura-se em três etapas/fases, que são: (a)

Problematização Inicial (PI): etapa que visa levantar as concepções prévias dos estudantes a respeito do assunto a ser tratado em aula; (b) Organização do Conhecimento (OC): etapa em que deve ocorrer a introdução de conhecimentos científicos e o desenvolvimento de conhecimentos novos para os estudantes; e (c) Aplicação do Conhecimento (AC): etapa que destina-se à verificação da aprendizagem, sobretudo, com a utilização dos conhecimentos construídos pelos estudantes para interpretar as situações problematizadas inicialmente; e parte de uma temática central que perpassa por todas as atividades de aprendizagem que compõem uma sequência didática $(\mathrm{SD})$.

${ }^{5} \mathrm{O}$ Ciclo de Kolb é composto pelas etapas: (a) Experiência concreta: etapa que busca investigar os conhecimentos prévios dos estudantes provindos das experiências vividas por cada um; (b) Observação reflexiva: etapa que proporciona o confronto das experiências concretas de cada estudante com as experiências concretas dos outros estudantes; (c) Conceituação abstrata: etapa da sistematização do conhecimento novo. Nela, os estudantes são levados a estabelecerem as ligações possíveis entre os diversos conhecimentos anteriores e os conhecimentos novos; (d) Experimentação ativa: etapa da verificação da aprendizagem.

${ }^{6}$ Disponível em: <http://phet.colorado.edu/en/simulation/reversible-reactions > Acesso em: 10 maio 2015.
} 
Bulegon, A. M.; Tarouco, L. M. R.

Quadro 2. Disposição das atividades com os OAs na metodologia dos TMP e Ciclo de Kolb

\begin{tabular}{|c|c|c|c|c|}
\hline TMP & Ciclo de Kolb & Tipo de OA & Assunto & Objetivos dos OA \\
\hline PI & $\begin{array}{l}\text { Experiência } \\
\text { concreta }\end{array}$ & Questionário & $\begin{array}{l}\text { - Introdução à } \\
\text { Termodinâmica }\end{array}$ & $\begin{array}{l}\text { Relatar/relembrar } \\
\text { experiências concretas de } \\
\text { suas vidas. }\end{array}$ \\
\hline OC & $\begin{array}{c}\text { Observação } \\
\text { reflexiva } \\
\text { Conceitualização } \\
\text { abstrata }\end{array}$ & $\begin{array}{l}\text { Texto, vídeo, } \\
\text { simulação }\end{array}$ & $\begin{array}{l}\text { - Máquinas térmicas e } \\
\text { Revolução Industrial; } \\
\text { - Trabalho termodinâmico; } \\
\text { - Conservação de } \\
\text { energia - Primeira Lei da } \\
\text { Termodinâmica; } \\
\text { - Entropia - Segunda Lei } \\
\text { da Termodinâmica }\end{array}$ & Observação e reflexão \\
\hline $\mathrm{AC}$ & $\begin{array}{l}\text { Experimentação } \\
\text { ativa }\end{array}$ & $\begin{array}{l}\text { Texto, vídeo, } \\
\text { simulação }\end{array}$ & - Bicombustíveis & $\begin{array}{l}\text { Gerar a experimentação } \\
\text { ativa e verificação da } \\
\text { aprendizagem. }\end{array}$ \\
\hline
\end{tabular}

Fonte: elaborado pelas autoras.

Quadro 3. Atividade de aprendizagem com vídeo

\begin{tabular}{|l|l|}
\hline Atividade & $\begin{array}{l}\text { - Agora que você já sabe o que são máquinas térmicas, acesse o link <https://www. } \\
\text { youtube.com/watch?v=yda4STR1Pe4> e verifique o funcionamento de uma máquina } \\
\text { a vapor; }\end{array}$ \\
- Em seguida, acesse o link <https://www.youtube.com/watch?v=emRxXykWB3Y> \\
e veja o funcionamento de um motor de combustão interna (motor automotivo); \\
- A partir do que foi visto nos vídeos, responda: \\
1) O motor do automóvel de hoje tem algo em comum com a máquina a vapor? \\
2) Como ocorre o movimento nos motores dos automóveis e das máquinas a vapor? \\
Quem produz esse movimento? \\
- Poste suas respostas a essas perguntas no fórum “Máquinas térmicas”.
\end{tabular}

Fonte: elaborado pelas autoras.

$\mathrm{Na} \mathrm{AC}$, os objetos de aprendizagem (vídeo, texto, simulação) foram utilizados para gerar a experimentação ativa do conhecimento novo e verificar a aprendizagem. Nesta etapa, fez-se uso de um texto sobre o tema Biocombustíveis ${ }^{7}$; um vídeo (parte 1 e 2) sobre a produção de Etanol no Brasil ${ }^{8}$ e um teste. Esse último tem como objetivo verificar a aprendizagem sobre Termodinâmica.

7 O texto utilizado foi: ÁLCOOL ou gasolina?: tanto faz. Veja, São Paulo, n. 1.756, p. 68, jun. 2002.

8 Disponível em: <http:/ /www.youtube.com/watch?v=r94Gubjv7Ug>. Acesso em: 06 maio 2015. 
As atividades de aprendizagem no Moodle incluíram o uso das ferramentas fórum e chat para interação. Ao participar de forma interativa, o estudante compromete-se ativamente nas discussões, facilitando a construção do conhecimento que “[...] ocorre quando os estudantes exploram estas questões, tomam posição, discutem as posições sob uma forma argumentativa, reavaliam e refletem sobre suas posições" (TAROUCO et al., 2008, p. 6).

\section{b) Contexto da pesquisa}

A pesquisa foi desenvolvida em uma Escola Estadual de Ensino Médio de uma cidade do interior do Rio Grande do Sul, com estudantes separados em turmas experimentais e de controle, totalizando 220 estudantes com idade entre 15 e 18 anos, com nível heterogêneo de conhecimento, matriculados na $2^{\mathrm{a}}$ série do Ensino Médio. A carga horária dessa disciplina era de três horas-aula semanais. O módulo digital trabalhado com os estudantes das turmas experimentais ocupou dez horas-aula. Essas aulas foram desenvolvidas de forma presencial, no Laboratório de Informática da escola, e eram parte da carga horária da disciplina de Física. O Laboratório de Informática da escola era equipado com um computador com acesso à Internet para cada estudante, e, para a professora, projetor multimídia, quadro branco e caneta. A turma de controle teve aulas em sala convencional (com diversos professores), e o tema Termodinâmica foi trabalhado com uso de livro didático, quadro e giz, de maneira expositiva e dialogada, sem o uso do computador.

\section{c) Coleta e análise dos dados}

Na coleta de dados, foram utilizados questionários no início (questionário 1, com quatro questões) e no final (questionário 2, com seis questões) da implementação do módulo. Os estudantes foram numerados por E1, E2, E3, assim por diante, para preservar sua identidade. O questionário 1 teve como objetivo verificar a existência de concepções prévias, na estrutura cognitiva dos estudantes, necessárias e adequadas para que o novo conhecimento pudesse ser ancorado e facilitar a ocorrência do desenvolvimento do pensamento crítico. Além de verificar a presença dos indicadores de pensamento crítico propostos por Newman, Webb e Cochrane (1995). O questionário 2 objetivou verificar o desenvolvimento do pensamento crítico, após o desenvolvimento de atividades de aprendizagem com o uso de OA. No final do desenvolvimento das atividades de aprendizagem que compõem o $\mathrm{MD}$, aplicou-se o questionário 3, para verificar se os tipos de OA utilizados nas atividades de aprendizagem são materiais potencialmente significativos e contribuem para o desenvolvimento do pensamento crítico.

As respostas dos questionários 1 e 2 foram organizadas, analisadas e marcadas de acordo com a constatação da presença dos indicadores de pensamento crítico de Newman, Webb e Cochrane (1995). Os índices de pensamento crítico, derivados dessa análise, não se limitam a afirmativas verdadeiras ou falsas no processo de construção do conhecimento, mas são marcados em pares de indicadores positivos (+) e negativos (-), e quantificados de acordo com a forma proposta por Medina (2004), assim descrita: Ind $=\left(\operatorname{Ind}^{+}-\mathrm{Ind}^{-}\right) /\left(\mathrm{Ind}^{+}+\mathrm{Ind}\right)$, onde $\left(\mathrm{Ind}^{+}\right)$são os totais de indicadores positivos e (Ind) os totais de indicadores negativos. Entende-se por $\mathrm{Ind}^{+}$aqueles dados que indicam a presença do indicador de pensamento crítico de Newman, Webb e Cochrane (1995), e por Ind' aqueles que não apresentam a presença do indicador. Por exemplo, para a questão 2 (Quando o ar é comprimido rapidamente, por que sua temperatura aumenta?) do questionário 1, uma das respostas e o resultado de sua análise são apresentados no Quadro 4, onde são analisados os indicadores nela constatados. 
Bulegon, A. M.; Tarouco, L. M. R.

Quadro 4. Exemplo de análise dos indicadores de PC de Newman, Webb e Cochrane (1995)

\begin{tabular}{|c|l|c|c|c|c|c|c|c|c|c|c|}
\hline Estudante & $\begin{array}{c}\text { Indicadores de Pensamento } \\
\text { Crítico de Newman } \\
\text { e colaboradores }\end{array}$ & $\mathbf{C} / \mathbf{E}$ & $\mathbf{N}$ & $\mathbf{R}$ & $\mathbf{I}$ & $\mathbf{A} / \mathbf{C}$ & $\mathbf{A}$ & $\mathbf{A} / \mathbf{I}$ & $\mathbf{J}$ & $\mathbf{U} / \mathbf{P}$ & $\mathbf{E X}$ \\
\hline R10 & $\begin{array}{l}\text { Por causa da pressão que causa nas } \\
\text { moléculas, fazendo, assim, elas se } \\
\text { agitarem mais rapidamente. }\end{array}$ & + & - & - & + & - & + & + & + & - & + \\
\hline R34 & Por causa do choque das moléculas. & - & - & - & + & - & + & - & - & - & - \\
\hline R21 & Não sei. & - & - & - & - & - & - & - & - & - & - \\
\hline R18 & $\begin{array}{l}\text { Por causa da agitação das moléculas } \\
\text { (choque entre as moléculas). }\end{array}$ & - & - & - & + & - & + & - & - & - & - \\
\hline
\end{tabular}

Fonte: elaborado pelas autoras.

Os índices encontrados dessa forma ficam limitados em uma escala de $-1 \mathrm{a}+1$, onde -1 representa um índice de ausência de pensamento crítico e +1 um pensamento crítico.

\section{Resultados e discussões}

Os resultados obtidos no desenvolvimento das atividades de aprendizagem que compõem o módulo Termodinâmica são apresentados e comentados a seguir. Segundo Jonassen (1996a), um estudante desenvolveu a habilidade do pensamento crítico quando é capaz de: identificar as informações relevantes de um problema, buscar relações de causalidade, reconhecer padrões e falácias, fazer comparações e interligar ideias. Os indicadores de Newman, Webb e Cochrane (1995) relacionados com essas habilidades são: Relevância (R), Importância (I), Ambiguidades (A), Justificativa (J), Utilidade Prática-Avaliação do conhecimento (U/P) e Extensão da Compreensão (EX). Esses indicadores puderam ser investigados nos resultados obtidos da aplicação dos questionários 1 e 2 no grupo experimental e no grupo de controle. Para o grupo experimental, a variação dos índices de indicadores de pensamento crítico (IPC) medido pelos questionários 1 (início do trabalho) e 2 (fim do trabalho) é apresentada na Tabela 1.

A análise desses dados mostra que houve melhora em todos os indicadores de pensamento crítico, tal como pode ser observado no Gráfico 1. Observa-se que o IGPC (índice geral de pensamento crítico) do questionário 1 (-0,40927) é menor do que o do questionário 2 $(-0,04466)$. Essa informação aponta que, após o estudo com o uso dos OA, houve um incremento positivo $(18,23 \%)$ no desenvolvimento do pensamento crítico dos estudantes.

A maior variação nos índices de pensamento crítico foi verificada no indicador Conhecimento/Experiência $(\mathrm{C} / \mathrm{E})$ (variação no índice de $0,8187=40,95 \%$ ). A presença acentuada desse indicador demonstra que os estudantes recorrem a experiências pessoais e/ou ao material do curso na busca de soluções dos problemas propostos, ou seja, usam as informações para resolver um problema e fazem uso do conhecimento abstrato para resolver situações do cotidiano. 
Tabela 1. Variação dos índices de indicadores de pensamento crítico do Grupo experimental

\begin{tabular}{|c|c|c|c|c|c|}
\hline Indicadores & & $\begin{array}{c}\text { Questionário } \\
1\end{array}$ & $\begin{array}{c}\text { Questionário } \\
2\end{array}$ & $\begin{array}{c}\text { Variação de IPC } \\
(\mathrm{Q} 2-\mathrm{Q} 1)\end{array}$ & $\begin{array}{c}\text { \% de variação } \\
\text { do IPC }\end{array}$ \\
\hline Conhecimento/Experiência & $\mathrm{C} / \mathrm{E}$ & $-0,4394$ & 0,3793 & 0,8187 & $40,95 \%$ \\
\hline Novidade & $\mathrm{N}$ & $-0,5769$ & $-0,44$ & 0,1369 & $6,84 \%$ \\
\hline Relevância & $\mathrm{R}$ & $-0,4102$ & $-0,2068$ & 0,2034 & $10,17 \%$ \\
\hline Importância & I & $-0,5063$ & $-0,1494$ & 0,3569 & $17,84 \%$ \\
\hline Avaliação Crítica & $\mathrm{A} / \mathrm{C}$ & $-0,4935$ & $-0,0543$ & 0,4392 & $21,96 \%$ \\
\hline Ambiguidades & A & $-0,0256$ & 0,5243 & 0,5499 & $27,49 \%$ \\
\hline Associação de Ideias & $\mathrm{A} / \mathrm{I}$ & $-0,3333$ & $-0,1474$ & 0,1859 & $9,29 \%$ \\
\hline Justificativa & $\mathrm{J}$ & $-0,2948$ & 0,0857 & 0,3805 & $19,02 \%$ \\
\hline Utilidade Prática & $\mathrm{U} / \mathrm{P}$ & $-0,5384$ & $-0,3218$ & 0,2166 & $10,83 \%$ \\
\hline Extensão da Compreensão & EX & $-0,4743$ & $-0,1162$ & 0,3581 & $17,90 \%$ \\
\hline IGPC & & $-0,40927$ & $-0,04466$ & 0,36461 & $18,23 \%$ \\
\hline
\end{tabular}

Fonte: elaborado pelas autoras.

Gráfico 1. Variação dos índices de indicadores de pensamento crítico do Grupo experimental

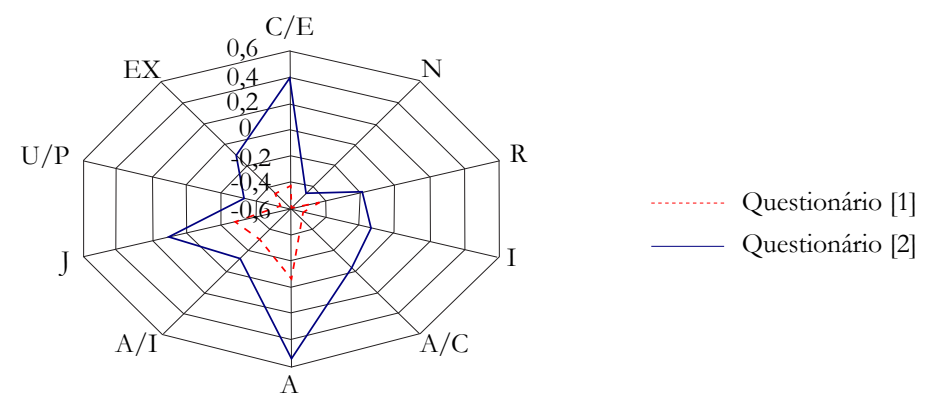

Fonte: elaborado pelas autoras.

Variações menos expressivas, mas também significativas, são as apresentadas nos indicadores Ambiguidade (27,49\%) e Avaliação Crítica (21,96\%). O indicador Ambiguidade chama-nos a atenção, pois, ao compararmos as respostas dos estudantes do questionário 1 para esse indicador, o número de respostas positivas foi semelhante ao número de respostas negativas. Diante disso, a variação para esse indicador evidencia que os estudantes responderam aos questionamentos de forma mais clara, discutindo-as e procurando esclarecê-las.

A variação do índice no indicador Avaliação Crítica demonstra que os estudantes passaram a refletir sobre suas próprias contribuições ou de outras pessoas, e estão abertos às críticas. As atividades de aprendizagem que proporcionaram, aos estudantes, essas habilidades 
Bulegon, A. M.; Tarouco, L. M. R.

fizeram uso de OA do tipo simulação, texto e vídeo. Em todas essas atividades, os estudantes deveriam responder a questões problematizadoras com base nas informações obtidas nos OA. Esses OA possibilitaram, aos estudantes, realizar um resgate de suas concepções prévias, fazer uso adequado de modelos explicativos e compreender as diferenças entre os tipos de variáveis envolvidas, bem como suas relações. Uma análise mais detalhada desses índices nos remete à construção de outra tabela (Tabela 2) e de um outro gráfico (Gráfico 2), que demonstram a variação dos indicadores de pensamento crítico nos estudantes do grupo experimental.

Tabela 2. Variação dos indicadores de Pensamento Crítico nos estudantes do grupo experimental

\begin{tabular}{lcccccccccc}
\hline Indicadores & C/E & $\mathbf{N}$ & $\mathbf{R}$ & $\mathbf{I}$ & $\mathbf{A} / \mathbf{C}$ & $\mathbf{A}$ & $\mathbf{A} / \mathbf{I}$ & $\mathbf{J}$ & $\mathbf{U} / \mathbf{P}$ & $\mathbf{E X}$ \\
\hline Variação & 0,8187 & 0,1369 & 0,2034 & 0,3569 & 0,4392 & 0,5499 & 0,1859 & 0,3805 & 0,2166 & 0,3581 \\
\hline
\end{tabular}

Fonte: elaborado pelas autoras.

Gráfico 2. Variação dos indicadores de pensamento crítico nos estudantes do grupo experimental

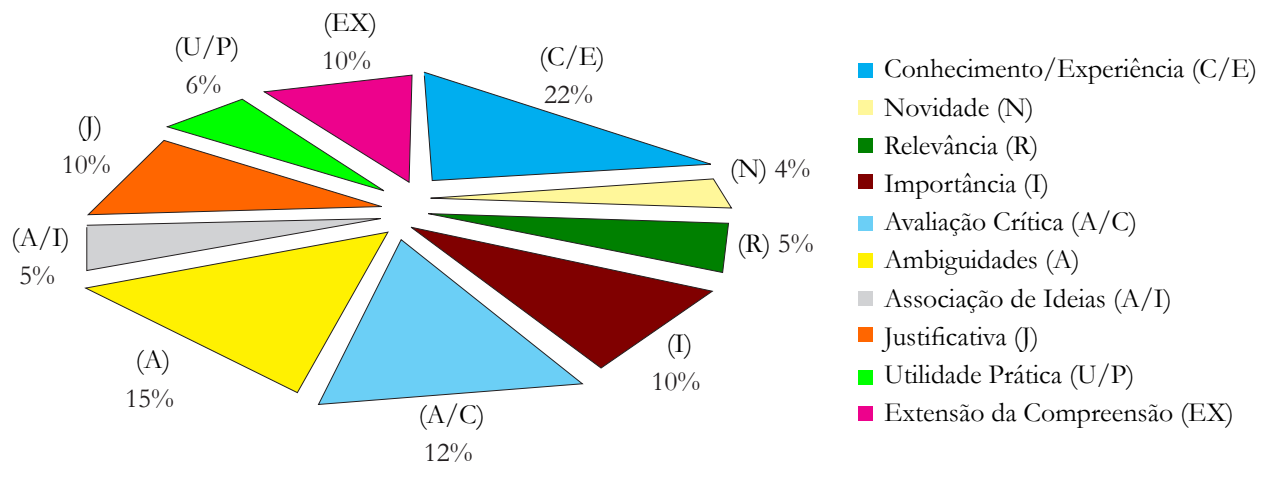

Fonte: elaborado pelas autoras.

A análise desse gráfico confirma a maior evolução para os indicadores Conhecimento/ Experiência (22\%), Ambiguidade (15\%) e Avaliação Crítica (12\%). Os indicadores Justificativa, Importância e Extensão da Compreensão obtiveram um percentual de $10 \%$, o que sinaliza o desenvolvimento de importantes habilidades do pensamento crítico relacionadas: à emissão de juízos próprios; ao fornecimento de provas ou exemplos para justificar suas soluções; à defesa de 
suas posições com argumentação e embasamento; à identificação de relações importantes entre o conteúdo e o problema, além da interpretação de observações do cotidiano. Os indicadores Relevância (R) e Utilidade Prática $(\mathrm{U} / \mathrm{P})$ obtiveram um percentual de $6 \%$ e foram seguidos, de perto, pelo indicador Associação de Ideias (A/I), que obteve um percentual de 5\%. Esses últimos indicadores demonstram que os estudantes forneciam depoimentos relevantes nos questionamentos e procuravam relacionar possíveis soluções a situações familiares. Além disso, associavam fatos e relacionavam novos dados a partir das informações coletadas, como pode ser percebido nas respostas à questão 6 (Após tudo o que foi estudado até o momento, qual sua opinião sobre o uso de bicombustíveis como fonte de energia nos veículos?) do questionário 2 :

\begin{abstract}
Acredito que, apesar de ser uma alternativa onde o rendimento é menor, ainda assim é a melhor escolba, pois os bicombustiveis são menos poluentes que os combustiveis fósseis, e certamente com o tempo os cientistas descobrirão uma forma de obter o mesmo rendimento entre eles. [E2]

Em minha opinião, os bicombustiveis trazem grandes benefícios para o meio ambiente epara a economia. Sendo assim, é viável que se adote essa forma de obter energia. [E3] O uso de bicombustivel foi uma forma de diminuir a emissão de poluentes na atmosfera, além de ser uma fonte renovável. [E4]

Sou a favor. Além de terem um custo mais barato, tanto na compra quanto na produção, poluem bem menos que a gasolina e o diesel. [E17]
\end{abstract}

A atividade de aprendizagem usada para ensejar este tipo de desenvolvimento cognitivo fez uso de um OA do tipo vídeo, que trazia informações a respeito da produção de Etanol no Brasil. Após assistir ao vídeo, eles discutiram com seus colegas, em um fórum, as informações presentes nele e pesquisaram sobre as consequências que o uso de bicombustíveis traria para a sociedade do séc. XXI.

A menor variação no índice de pensamento crítico, nesta comparação, foi o indicador Novidade (N), com uma variação de 4\%. Apesar de as atividades de aprendizagem com o uso dos OA serem, em sua maioria, investigativas e previamente elaboradas e disponibilizadas aos estudantes, a análise desse índice demonstra o comportamento passivo de muitos estudantes diante das atividades de aula, pois, geralmente, esperavam pelas instruções da professora para iniciar as atividades de aprendizagem. Possivelmente, esse comportamento tenha ocorrido devido ao caráter de novidade que o trabalho no Moodle proporcionou a todos, pois os estudantes não haviam tido aulas nesse modelo e nem com o uso de um ambiente virtual.

Os índices discutidos nas páginas anteriores, quando comparados ao grupo de controle, apontam variações e podem ser identificados na Tabela 3 e no G3. Esses mostram o resultado geral dos índices de todos os indicadores para o questionário 2 do grupo experimental e de controle.

Os dados da Tabela 3 mostram que houve um incremento positivo de $28 \%$ no desenvolvimento do pensamento crítico dos estudantes do grupo experimental em relação ao grupo de controle analisado. Esse dado foi obtido pelo IGPC (índice geral de pensamento crítico). O Gráfico 3, permite constatar a variação em todos os indicadores de pensamento crítico nos estudantes dos grupos experimental e de controle. 
Bulegon, A. M.; Tarouco, L. M. R.

Tabela 3. Comparativo entre a variação dos índices de Pensamento Crítico (IPC) do Grupo experimental e do Grupo de controle após desenvolvimento do conteúdo Termodinâmica

\begin{tabular}{lccccc}
\hline \multicolumn{1}{c}{ Indicadores } & & $\begin{array}{c}\text { Grupo } \\
\text { Experimental }\end{array}$ & $\begin{array}{c}\text { Grupo de } \\
\text { controle }\end{array}$ & $\begin{array}{c}\text { Variação de IPC } \\
\text { (GE - GC2) }\end{array}$ & $\begin{array}{c}\text { Percentual de } \\
\text { variação do IPC }\end{array}$ \\
\hline Conhecimento/Experiência & $\mathrm{C} / \mathrm{E}$ & 0,3793 & $-0,3333$ & 0,7126 & $35,63 \%$ \\
Novidade & $\mathrm{N}$ & $-0,4425$ & $-0,5897$ & 0,1472 & $7,36 \%$ \\
Relevância & $\mathrm{R}$ & $-0,2068$ & $-0,6000$ & 0,3932 & $19,66 \%$ \\
Importância & $\mathrm{I}$ & $-0,1498$ & $-0,6000$ & 0,4502 & $22,51 \%$ \\
Avaliação Crítica & $\mathrm{A} / \mathrm{C}$ & $-0,0543$ & $-0,7435$ & 0,6892 & $34,46 \%$ \\
Ambiguidades & $\mathrm{A}$ & 0,5243 & $-0,4820$ & 1,0063 & $50,31 \%$ \\
Associação de Ideias & $\mathrm{A} / \mathrm{I}$ & $-0,1474$ & $-0,7384$ & 0,5910 & $29,55 \%$ \\
Justificativa & $\mathrm{J}$ & $-0,0857$ & $-0,5128$ & 0,4271 & $21,35 \%$ \\
Utilidade Prática & $\mathrm{U} / \mathrm{P}$ & $-0,3218$ & $-0,8102$ & 0,4884 & $24,42 \%$ \\
Extensão da Compreensão & $\mathrm{EX}$ & $-0,1162$ & $-0,8102$ & 0,6940 & $34,7 \%$ \\
IGPC & & $-0,06209$ & $-0,62201$ & 0,55992 & $28,00 \%$ \\
& & & & &
\end{tabular}

Fonte: elaborado pelas autoras.

Gráfico 3. Comparativo entre a variação dos índices de pensamento crítico do Grupo experimental e do Grupo de controle após desenvolvimento do conteúdo Termodinâmica

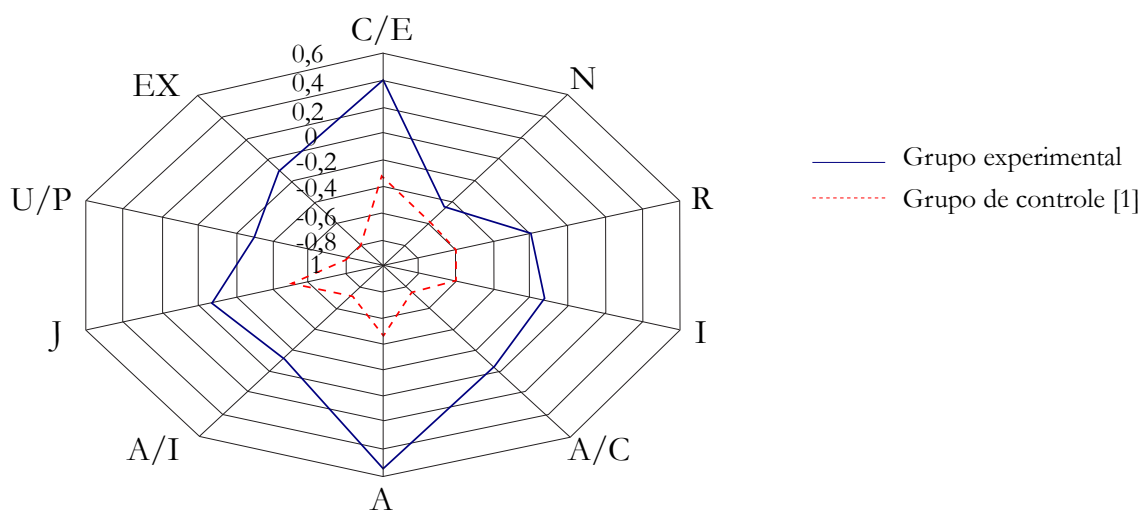

Fonte: elaborado pelas autoras.

A análise dos gráficos e tabelas acima nos mostra que, em ambos os grupos (experimental e de controle), há evidências do desenvolvimento do pensamento crítico. Entretanto, observa-se que os índices de pensamento crítico são superiores para o grupo experimental em todos os aspectos avaliados. Uma análise dessa variação mostra uma diferença acentuada nos índices de pensamento crítico no Grupo experimental em todos os indicadores em relação ao grupo de controle. Houve, de forma significativa, índices altamente positivos, como os indi- 
cadores: Ambiguidades (50,31\%), o que denota clareza nas respostas; Conhecimento/Experiência (35,63\%), evidenciando o uso de conhecimentos prévios, experiências pessoais ou referência ao material do curso na resolução dos problemas propostos. O indicador Avaliação Crítica $(34,46 \%)$ assinala que os estudantes avaliam/contribuem de forma crítica e estão abertos a discussões. O indicador Associação de Ideias/Interpretação (29,55\%) aponta que os estudantes relacionam as informações, comparam e geram novos dados a partir de informações coletadas. O indicador Extensão da compreensão, com um índice de 34,7\%, mostra que os estudantes emitem juízos próprios e tomam decisões que requeiram compreensão do conhecimento. Além desses indicadores, podemos observar que também houve o desenvolvimento de outros, como: Relevância, Justificativa, Importância, Utilidade Prática. São índices menores, mas não menos expressivos, pois de $19 \%$ a $24 \%$ das respostas apresentaram indicadores positivos, o que denota uma presença significativa de desenvolvimento de pensamento crítico.

A análise realizada sobre a contribuição dos OA no desenvolvimento do pensamento crítico, em aspectos relacionados aos indicadores, apontou que aqueles aspectos onde a variação foi menor não eram explicitamente destacados nos OA. Isso permite elicitar duas conclusões importantes: (1) o uso de OA contribui para o desenvolvimento do pensamento crítico de modo geral, embora todos os indicadores não fossem explicitamente objeto de atenção no projeto do $\mathrm{OA}$; (2) os OA devem, intencionalmente, instigar o desenvolvimento do pensamento crítico em todos os aspectos, tal como elicitado no conjunto de indicadores de Newman, Webb e Cochrane (1995) ou em qualquer outro conjunto de indicadores de pensamento crítico.

\section{Considerações finais sobre os resultados observados tendo em vista os tipos de objetos de aprendizagem utilizados}

Esta pesquisa permitiu constatar que realizar uma atividade de aprendizagem com o uso dos OA do tipo simulação, por mais simples que seja, traz uma renovação, consegue despertar o mais pacato dos estudantes e o faz ver a Física sobre outra ótica. Os OA desse tipo geram aspectos e fatores que vão além da aprendizagem conceitual, como a observação e a atenção, buscam o envolvimento do aprendiz; geram criatividade, pois há hipóteses a serem levantadas e testes a serem realizados; geram confiança e satisfação, resultante da percepção do alcance das metas traçadas, e levam o aprendiz a envolver-se mais em sua aprendizagem. Ampliam, dessa forma, o grau de aplicação de seu conhecimento e a criação de novas situações de aprendizagem.

As atividades de aprendizagem com o uso dos OA do tipo vídeo permitiram, aos estudantes, o desenvolvimento de algumas habilidades importantes para o desenvolvimento do pensamento crítico, como: conhecimento/experiência, associação de ideias, análise crítica, relevância, justificativa, importância e utilidade prática. Além disso, permitiram, aos estudantes, rever o conteúdo estudado fora do horário da aula, em suas casas e/ou em outros ambientes que não o da sala de aula presencial. Isso ocasionou a ampliação do tempo dedicado, pelo estudante, para a disciplina de Física, e o estudo para além dos muros escolares foi estimulado.

A ferramenta fórum, do Moodle, estimulou a reflexão e análise dos estudantes sobre seu conhecimento. Com essa ferramenta, o estudante podia fazer comparações e concentrarse mais nos objetivos da aula, além de discutir e trocar ideias com os demais colegas sobre os assuntos estudados. A participação e a interação no fórum permitiram a troca de saberes entre 
os estudantes, o que potencializou a aprendizagem, pois podiam ver as respostas dos colegas, refletir sobre elas, tirar conclusões e comentá-las. As habilidades de pensamento crítico desenvolvidas com essa ferramenta são: a realização de uma avaliação crítica sobre seu conhecimento/ experiência; a interpretação e associação de fatos/ideias e a discussão de sua utilidade prática; a justificativa de soluções ou julgamentos próprios; a qualidade de suas contribuições, com depoimentos relevantes e escrita de ideias de forma mais clara.

Este trabalho partiu da premissa de que os OA deveriam estar inseridos em um modelo metodológico, e que deveriam ser interativos, contextualizados e colaborativos, como características básicas. $\mathrm{O}$ uso de $\mathrm{OA}$ interativos e contextualizados, nas atividades de aprendizagem, proporcionou, aos estudantes, verificarem as variáveis envolvidas e suas relações na resolução dos problemas. Com isso, os estudantes puderam desenvolver uma aprendizagem não apenas para resolver problemas escolares, mas, também, problemas cotidianos. Além disso, a reflexão, proporcionada pela interação com os $\mathrm{OA}$, permitiu, aos estudantes, desenvolverem um papel ativo, corresponsabilizando-se pelos rumos, profundidade e significado de seu aprendizado, levando-os ao desenvolvimento do pensamento crítico.

Ressalta-se que as atividades de ensino com o uso de OA despertam interesse nos estudantes, entretanto, se não forem trabalhadas a partir de roteiros elaborados segundo uma proposta metodológica apropriada, podem não promover o desenvolvimento do pensamento crítico. As atividades de aprendizagem com o uso de OA, desenvolvidas neste trabalho e inseridas no modelo metodológico dos TMP e organizadas de acordo com o Ciclo de Kolb, provocaram: uma intensa participação, interação, colaboração, exercício de criatividade e construção do conhecimento nos estudantes envolvidos. Com isso, eles assumiram a postura de colaborador e autor de seu próprio conhecimento, evidenciando o desenvolvimento do pensamento crítico e criativo. O software de autoria eXe Learning permitiu criar, desenvolver e publicar os conteúdos no MD, organizando os conteúdos e possibilitando agregar diversas mídias e potencializar as possibilidades pedagógicas de interação.

Apesar da complexidade inicial de se trabalhar com os OA, esses podem ser recursos valiosos para promover uma maior participação dos estudantes nas unidades de aprendizagem, e tornar o ensino estimulante e melhorar a aprendizagem e a retenção significativa. As dificuldades enfrentadas relativas ao uso da tecnologia foram superadas na medida em que o processo de uso da mesma foi compartilhado entre todos. Isso fez com que a aprendizagem ocorresse de forma mais rápida e se tornasse significativa. Eles mostram-se, também, adequados para o ensino e a aprendizagem de vários conteúdos conceituais da Física (conceitos, leis e fórmulas) e, em especial, tópicos de Termodinâmica.

Temos consciência de que toda a mudança de paradigma não é um processo rápido e linear, pois mexe com a estrutura, com a cultura e com os hábitos das pessoas. O comprometimento com o novo depende, em primeiro lugar, do convencimento de que a nova situação vai nos trazer resultados mais significativos que os obtidos em situações anteriores. Este trabalho investigativo permitiu constatar e avaliar a possibilidade e intensidade do desenvolvimento de pensamento crítico mediante o uso de OA especialmente construídos, porém, não encerra-se neste momento. Percebem-se diversificadas oportunidades para dar continuidade à pesquisa até aqui realizada, e alguns desdobramentos são apontados, como: mostrar aos professores, dos diversos níveis de ensino, as possibilidades de trabalhar com os OA e os AVA para o desenvolvimento do Pensamento Crítico. 


\section{Agradecimentos}

Agradecemos a Prof ${ }^{a}$ Dr $^{a}$ Eliane Ângela Veit, do Instituto de Física da UFRGS, por sua valiosa contribuição como coorientadora do trabalho de tese que originou este artigo.

\section{Referências}

A FÍSICA NA ESCOLA. São Paulo: Sociedade Brasileira de Física, [19--?]. ISSN 1983-6430. (Consulta 2004-2006).

ASSIS, L. P. Ensino de física em escolas do município de Niterói: concepções de professores e procedimentos didáticos. In: REUNIÃO ANUAL DA ASSOCIAÇÃO NACIONAL DE PÓS-GRADUAÇÃO E PESQUISA EM EDUCAÇÃO, 24., 2001, Caxambu.

Anais... Caxambu: UFMG: ANPED. Disponível em: <http://24reuniao.anped.org.br/ p0472470957102.doc >. Acesso em: 20 jul. 2010.

BECK, R. J. What are learning objects? Milwaukee: Center for International Education, 2001. Disponível em: <http://www4.uwm.edu/cie/research/conferences/LO_what.html>. Acesso em: 20 jul. 2010.

BRASIL. Lei $\mathrm{n}^{\circ} \mathbf{9 . 3 9 4}$ de 20 de dezembro de 1996. Estabelece as diretrizes e bases da educação nacional. Brasília, 1996. Disponível em: <http://portal.mec.gov.br/seed/arquivos/ pdf/tvescola/leis/lein9394.pdf>. Acesso em: 20 nov. 2011.

2000.

. Ministério da Educação. Parâmetros curriculares nacionais: introdução. Brasília,

CADERNO BRASILEIRO DE ENSINO DE FÍSICA. Florianópolis: Universidade Federal de Santa Catarina, 1984- . Trimestral. ISSN 1677-2334. (Consulta 2004-2009).

CIÊNCIA \& EDUCAÇÃO. Bauru: Universidade Estadual Paulista, 1994- . Trimestral. ISSN 1980-850X. (Consulta 2004-2009).

DAMASIO, F.; STEFFANI, M. H. A física nas séries iniciais $\left(2^{\mathrm{a}}\right.$ a $\left.5^{\mathrm{a}}\right)$ do ensino fundamental: desenvolvimento e aplicação de um programa visando a qualificação de professores. Revista Brasileira de Ensino de Física, São Paulo, v. 30, n. 4, p. 4503.1-4503.9, 2008. Disponível em: <http://www.sbfisica.org.br/rbef/pdf/304503.pdf>. Acesso em: 20 jul. 2010.

DELIZOICOV, D.; ANGOTTI, J. A. Física. São Paulo: Cortez, 1991.

ENNIS, R. H. A logical basis for measuring critical thinking skills. Educational

Leadership, Champaign, v. 43, n. 2, p. 44-48, 1985. Disponível em: <http://www.ascd.org/ ASCD/pdf/journals/ed_lead/el_198510_ennis.pdf>. Acesso em: 10 ago. 2010.

FIOLHAIS, C.; TRINDADE, J. Física no computador: o computador como uma ferramenta no ensino e na aprendizagem das ciências físicas. Revista Brasileira de Ensino de Física, São Paulo, v. 25, n. 3, p. 259-272, 2003. 
Bulegon, A. M.; Tarouco, L. M. R.

HALPERN, D. F. Teaching for critical thinking: helping college students develop the skills and dispositions of a critical thinker. New Directions for Teaching and Learning, San Francisco, v. 1999, n. 80, p. 69-74, 1999.

INVESTIGAÇÕES EM ENSINO DE CIÊNCIAS. Porto Alegre: Universidade Federal do Rio Grande do Sul, 1996- . Quadrimestral. ISSN 1518-9384. (Consulta 2005-2008).

JONASSEN, D. Computers in the classroom: mindtools for critical thinking. Englewood Cliffs: Simon \& Schuster, 1996a.

. O uso das novas tecnologias na educação a distância e a aprendizagem

construtivista. Em Aberto, Brasília, v. 16, n. 70, p. 80-88, abr/jun. 1996b. Disponível em:

<http://www.rbep.inep.gov.br/index.php/emaberto/article/viewFile/1054/956>. Acesso em: 10 mar. 2010.

KAYA, N. Critical fhinking skills of the students of university. 1997. Dissertation (PhD) - Graduate Scool of Health Sciences, Istanbul University, Istanbul, 1997.

KOLB, D.; FRY, R. Toward an applied theory of experiential learning. In: COOPER, C. L. Theories of group processes. London: John Wiley, 1975. p. 40-48.

MANDERNACH, B. J. et al. The role of instructor interactivity in promoting critical thinking in online and face-to-face classrooms. MERLOT Journal of Online Learning and Teaching, Long Beach, v. 5, n. 1, p. 49-62, 2009. Disponível em: < http://jolt.merlot. org/vol5no1/mandernach_0309.pdf>. Acesso em: 11 abr. 2010.

MEDINA, N. O. Avaliação do pensamento crítico em um cenário de escrita colaborativa. 2004. 100 f. Tese (Doutorado em Engenharia de Produção) - Universidade Federal de Santa Catarina, Florianópolis, 2004. Disponível em: <http://www6.ufrgs.br/ limc/escritacoletiva/pdf/avaliacao_do_pensamento.pdf>. Acesso em: 01 jun. 2010.

MINAYO, M. C. S.; SANCHES, O. Quantitativo-qualitativo: oposição ou complementaridade? Cadernos de Saúde Pública, Rio de Janeiro, v. 9, n. 3, p. 239-262, jul/set. 1993. Disponível em: < http://dx.doi.org/10.1590/S0102-311X1993000300002>. Acesso em: 01 jun. 2010.

NEWMAN, D. R.; WEBB, B.; COCHRANE, C. A content analysis method to measure critical thinking in face-to-face and computer supported group learning. Interpersonal Computing and Technology, Bloomington, v. 3, n. 2, p. 56-77, 1995. Disponível em: <http://www.helsinki.fi/science/optek/1995/n2/newman.txt>. Acesso em: 30 abr. 2010.

REVISTA BRASILEIRA DE ENSINO DE FÍSICA. São Paulo: Sociedade Brasileira de Física, 1979- . ISSN 1086 - 9126. (Consulta 2004-2009).

ROSA, P. R. S. O uso de computadores no ensino de física. Parte I: Potencialidade e uso real. Revista Brasileira de Ensino de Física, São Paulo, v. 17, n. 2, p. 182-195, 1995.

SALIÉS, T. G. Produzindo textos acadêmicos em língua estrangeira: o que está em jogo?

Caderno de Letras, Rio de Janeiro, n. 24, p. 13-27, 2008. 
SCHON, D. A. Educando o profissional reflexivo: um novo design para o ensino e a aprendizagem. Porto Alegre: Artes Médicas Sul, 2000.

SENDAG, S.; ODABASI, H. F. Effects of an online problem based learning course on content knowledge acquisition and critical thinking skills. Computers \& Education, New York, v. 53, n. 1, p. 132-141, 2009.

SUMNER, W. Folkways: a study of the sociological importance of usages, manners, customs, mores, and morals. Boston: Ginn, c1906. Disponível em: < http:/ /www.gutenberg. org/ebooks/24253>. Acesso em: 08 maio 2015.

TAROUCO, L. M. R. et al. Fatores que afetam a performance da comunicação mediada por computador. RENOTE: revista novas tecnologias na educação, Porto Alegre, v. 6, n. 2 , dez/2008. Disponível em: <http://seer.ufrgs.br/renote/article/view/14694>. Acesso em: 11 fev. 2011.

Objetos de aprendizagem para M-learning. In: CONGRESSO NACIONAL

DE TECNOLOGIA DA INFORMAÇÃO E COMUNICAÇÃO, 2004, Florianópolis.

Disponível em: <http://www.cinted.ufrgs.br/CESTA/objetosdeaprendizagem_sucesu.pdf $>$. Acesso em: 12 jan. 2010.

TRIVINÕS, A. N. S. Introdução à pesquisa em ciências sociais. São Paulo: Atlas, 1995.

VIEIRA, R. M.; VIEIRA, C. T. A formação inicial de professores e a didáctica das ciências como contexto de utilização do questionamento orientado para a promoção de capacidades de pensamento crítico. Revista Portuguesa de Educação, Braga, v. 16, n. 1, p. 231-252, 2003. Disponível em: <http://www.redalyc.org/articulo.oa?id=37416110>. Acesso em: 10 ago. 2011.

WELLER, M.; PEGLER, C.; MASON, R. Putting the pieces together: what working with learning objects means for the educator. [Edinburg, 2003]. Disponível em: $<$ http:/ / smartsheep.org/putting-the-pieces-together-what-working-with-learning-objects>. Acesso em: 06 maio 2015. 